raum selbst. ${ }^{10}$ Auch und vor allem aufgrund des umfangreichen Materials wird größtenteils auf eine Kommentierung und Interpretation der einzelnen Beiträge verzichtet, um in erster Linie das empirische Material des Monat in den Vordergrund zu stellen und von »selbst sprechen « zu lassen. Es geht hierbei um eine möglichst objektive Darstellung. Anschließend erfolgen eine kommentierende Zusammenfassung und das als Thesen formulierte "Analytische Resümee«, wo der Versuch unternommen wird, vor dem Hintergrund der aufgeworfenen Fragen eine Gesamteinschätzung der »Analyse des Nationalsozialismus« im Monat vorzunehmen; hierbei ließen sich inhaltliche Redundanzen nicht vermeiden.

\title{
1.3 Einführung zum Nationalsozialismus: Dr. Goebbels privat
}

Die erste Veröffentlichung in der Zeitschrift Der Monat, die sich direkt mit dem Nationalsozialismus beschäftigte, war die Rezension der Tagebücher Joseph Goebbels' (1897-1945) von Richard H. S. Crossmann, die unter dem Titel Dr. Goebels privat im ersten Heft veröffentlicht wurde. ${ }^{11}$ Die erste Eintragung im Tagebuch, das von Louis Lochner in einer "gekürzten Fassung ${ }^{12}$ herausgegeben wurde, stammte vom 21 . Januar 1942, wurde mit dem 23. Mai 1942 abgebrochen und dann von März bis Mai 1943 ohne große Unterbrechung vom Propagandaministers des >Dritten Reiches` weitergeführt.

Crossmann konstatierte zu Beginn seines Beitrages, dass Goebbels, der schon »vor der Machtergreifung « $^{13}$ mit dem Schreiben am Tagebuch anfing, zum Zeitpunkt der ersten Eintragung am 21. Januar 1942 in der Erwartung des militärischen Sieges ausschließlich an ein »Problem« dachte:

Das dringlichste war nach Coebbels' Meinung die Liquidierung des europäischen Judentums. Während es taktisch klug sein mochte, die Vernichtung der katholischen Kirche und der deutschen Oberschicht auf die Zeit nach dem Siege zu vertagen, mußte die Judenfrage sofort in Angriff genommen werden. Der Krieg gab die Möglichkeit, mit den Juden in einer Weise zu Verfahren, die in Friedenszeiten untunlich gewesen wäre. Coebbels war stolz auf seinen »Realismus « und seine elastische Haltung; aber in seinem Antisemitismus war er ganz und gar linientreuer Prinzipienreiter. Die Massenmor-

10 Die Veröffentlichungen, die primär aus Gründen der Auseinandersetzung mit der NS-Vergangenheit veröffentlicht wurden, nannte ich bereits in Kap. I.4, andererseits gehe ich darauf im Schlusskapitel ein. Insofern haben sie hier keine Bedeutung und werden nicht herangezogen.

11 R[ichard] H. S. Crossmann, Dr. Coebbels privat, in: Der Monat 1 (1948), H. 1, S. 90-93 (Rezension). Die Angaben zu dem Buch erfolgen hier - wie auch bei den folgenden Beiträgen - ausschließlich nach denen des Monat und werden nicht ergänzt. In der Regel erfolgten die Buchhinweise im Monat ohne ausdrückliche Angabe zum Erscheinungsjahr.

12 Ebd., S. 91. Crossmann wies auf S. 90 daraufhin, dass Coebbels jahrelang an seinem Tagebuch geschrieben hat und »ein Teil davon, beginnend mit den Monaten vor der Machtergreifung «, unter einem anderen Titel bereits während des Krieges veröffentlicht wurde, »allerdings mit zahlreichen Streichungen und `Verbesserungen««. Des Weiteren erklärte er, »daß um 1941 schon 23 Bände vorlagen«, und dass der »Verdreher der Wahrheit«, »der einzige nationalsozialistische Politiker [war], der Tag für Tag die geschichtlichen Ereignisse genau so zu Papier brachte, wie er sie sah« (S. 90 f.).

13 Ebd., S. 90. 
de mußten zu Ende gebracht werden, ohne Rücksicht auf den Widerstand der Kirchen und der Oberschicht. ${ }^{14}$

Crossmann stellte also fest, dass die nationalsozialistische Ermordung der europäischen Juden gekoppelt war an den Verlauf des Zweiten Weltkrieges und das gewissermaßen erst der Krieg die Möglichkeit eröffnete, den nationalsozialistischen Antisemitismus in die Tat umzusetzen, nämlich die Massenmorde in Gang zu bringen und vor allem auch »zu Ende« zu bringen. Und zwar, so der Rezensent, »ohne Rücksicht auf den Widerstand der Kirchen und der Oberschicht«. Gleichzeitig wurde von ihm die Ansicht vertreten, dass nach dem militärischen Erfolg sich die mörderische Energie des nationalsozialistischen Systems im Grunde genommen mit gleicher Qualität respektive Intensität gegen nicht jüdische Personen und Institutionen gerichtet hätte. Nämlich gegen die »katholischen Kirche« und die deutsche »Oberschicht«, weil dem Nationalsozialismus nach seiner Meinung aus ebendiesen Kreisen der deutschen Gesellschaft »Widerstand« gegen die »Massenmorde« an den europäischen Juden entgegengesetzt wurde. In diesem Zusammenhang fragte sich Crossmann:

Worin bestand die nächste Aufgabe nach der Ausschaltung der Juden? Coebbels' Antwort - die endgültige Vernichtung der bürgerlichen Klasse - zeigt, wie unrecht diejenigen marxistischen Theoretiker hatten, die den Nationalsozialismus lediglich als eine Tarnung des Monopolkapitalismus hinzustellen pflegten. In seiner Entschlossenheit, die Industriellen, die Richter, die Aristokratie und das Offizierskorps auszumerzen, war Coebbels ein echter Revolutionär. ${ }^{15}$

Goebbels' politisches und ideologisches Ressentiment fokussierte sich, vor dem Hintergrund der Vorstellung, dass die »bürgerliche Zivilisation« im »Sterben lag«, einerseits auf den sogenannten dekatenten Kapitalismus amerikanischer Provienenz und den »russischen Bolschewismus«, der für den nationalsozialistischen Propagandaminister eine »Perversion« darstellte. Andererseits galt »sein besonderer Haß« England, so Crossmann, weil nur dieses Land Goebbels' Ansicht nach in der Lage war, »eine neue soziale Ordnung auf arischer Grundlage zu errichten, die dem Dritten Reich gefährlich werden konnte«(S. 91). Der »Haß« von Goebbels auf England war auch darin begründet, so britische Politiker, »daß die Engländer, im Gegensatz zu den Amerikanern, gegen den Propagandakrieg unempfindlich seien« (ebd.). Denn für Goebbels stand fest:

Ein moralischer Zusammenbruch, wie wir ihn im November 1918 erlebten, kann in England, wenn überhaupt, nur mit großen Schwierigkeiten herbeigeführt werden. Wir sollten uns in dieser Beziehung keinen Illusionen hingeben und nicht Hoffnungen auf eine Kriegführung setzen, die früher einmal beim deutschen Volk zum Ziele führte, aber aller Wahrscheinlichkeit nach bei den Engländern niemals Erfolg haben wird. ${ }^{16}$

\footnotetext{
14 Ebd., S. 91.

15 Ebd.

16 Ebd.
} 
Im weiteren Verlauf seiner Besprechung legte Crossmann den thematischen Schwerpunkt auf den von Goebbels gezogenen Vergleich mit der Sowjetunion, der nämlich in seinem Tagebuch ausdrücklich weltanschauliche Reflexionen in Bezug auf den von ihm als »asiatisches Ungeheuer« apostrophierten Stalin und »dessen Totalitarismus und das ihm zur Verfügung stehende primitive Menschenmaterial« anstellte (ebd.). Und Crossmann schrieb hierzu:

Am 8. Mai 1943 berichtet er von längeren Ausführungen des Führers über die Stärke des Bolschewismus. Hitler sprach mit offenem Neid von den Vorteilen, die Stalin voraushatte, weil er »nicht durch den Widerstand einer Oberschicht gehemmt wird. Dieser Opposition hat er sich durch die Liquidierungen der letzten fünfundzwanzig Jahre entledigt«. Das gleiche gilt von den Kirchen.

Daran anschließend zitierte er einen weiteren Tagebucheintragung von Goebbels: »In dieser Beziehung hat es Stalin viel leichter als wir. Sein ganzes Volk spurt. Es unterliegt entweder der bolschewistischen Erziehung oder der bolschewistischen Knute [...]. Rußland beruht auf der festen geistigen Grundlage einer Ideologie - auch wenn es eine falsche ist. $\ll^{17}$

Vor diesem Hintergrund war der Propagandaminister Goebbels nach Einschätzung des Rezensenten ein »Nationalbolschewist«, der, um die Welt »in ein einziges Deutsches Reich umzuformen « - wie zum Beispiel in Frankreich oder Norwegen, so Crossmann, »die marxistische Philosophie von der Macht« benutzte (S. 92).

Aufgrund der oben angesprochenen Unterbrechung des goebbelsschen Tagebuches setzten die Eintragungen im März 1943 wieder ein, um dann bis Mai des selben Jahres quasi ohne Unterbrechung weitergeführt zu werden. Infolge der veränderten militärischen Situation - die deutsche 6. Armee unter der Führung von Feldmarschall Paulus hatte, nachdem sie in Stalingrad eingekesselt wurde, kapituliert; die deutschen Truppen in Nordafrika unter der Leitung von Generaloberst Hans-Jürgen von Arnim hatten in »Tunis« kapituliert; und in Deutschland sah man sich mit der »englisch-amerikanischen Luftoffensive « konfrontiert ${ }^{18}$ - veränderte sich, so Crossmann, der Blick und mithin die fast täglichen Eintragungen des Propagandaministers des $>$ Dritten Reiches $<$ radikal:

Seine Gedanken sind beherrscht von dem Drama eines heroischen Widerstandes unter seinem angebeteten Führer. Niederlagen scheinen Coebbels nur eine glänzende Gelegenheit, die Überlegenheit der SS über die Wehrmacht, der Partei über die Bürokratie, des Dritten Reiches über das Zweite zu zeigen. So wird er zum Robespierre der nationalsozialistischen Revolution, entschlossen, jeden militärischen Rückschlag durch eine noch unbarmherzigere Ausmerzung bürgerlicher Kleinmütigkeit wettzumachen. [...] Er spricht mit Verachtung von allen Kollegen und schont nur Hitler und Himmler, die seinen Fanatismus teilen. [...] Coebbels teilt Hitlers Haß gegen die Ceneräle und schürt 
ihn bewußt. Auf niemand in Deutschland ist wirklich Verlaß, ausgenommen die SS, die Partei und das einfache Volk. ${ }^{19}$

Die letzten Eintragungen im Tagebuch erfolgten zum Zeitpunkt der schweren alliierten Luftangriffe im Dezember des Jahres 1943. Goebbels hatte, wie Crossmann mitteilte, persönlich »die Feuerbekämpfung organisiert und den Pöbel mit einer glänzenden Rede im Titania-Palast aufgepeitscht« (ebd.). Trotz alledem zweifelte der Propagandaminister des >Dritten Reiches` angesichts der militärischen und politischen Situation immer mehr, so Crossmann, sodass er im Tagebuch vermehrt auf Eintragungen stieß, die »Anspielungen auf die Möglichkeit eines Separatfriedens« (ebd.) enthielten. Allerdings schrieb Goebbels selbst in einer der letzten Tagebucheintragungen hierzu: "Es ist so schwer, den Führer zu einer Entscheidung zu bewegen, und wir können natürlich nicht mit Moral allein gewinnen, wir müssen Waffen und Menschenmaterial haben. $\ll^{20}$

An dieser Stelle beendete Crossmann seine Ausführungen, Kommentare und Reflexionen in der Rezension zu Joseph Goebbels, Tagebücher. Vor diesem Hintergrund erfolgte dann eine Personenbeschreibung bzw. eine zusammenfassende Einschätzung über den »Politiker«, »Ideologen«, »Propagandaminister und »Privatmensch[en] « Goebbels. Nach Crossmanns Dafürhalten war der private Mensch Goebbels schwer zu greifen bzw. zu begreifen, weil in dessen Tagebucheintragungen auf das »Privatleben« im Grunde genommen nicht eingegangen wurde. Seiner Einschätzung nach war für Goebbels ebendieses Privatleben »lediglich eine Nebenerscheinung der Politik« wie »Essen und Trinken« (ebd.). Hier sah Crossmann einen Unterschied zu anderen NS-»Prominenten«, die in erster Linie daran dachten, »ihr Schäfchen ins Trockene zu bringen«, oder beispielsweise zu Göring, der ein »wohlgelaunter Raufbold« gewesen war (ebd.).

Crossmann fragte sich, ob Goebbels ein »Mephisto« war, »der kaltblütig eine Theorie vertrat, an die er selbst nicht glaubte, der selbst die Propagandalügen durchschaute, die er verbreitete?«(S. 92 f.) Genau diese Vorstellung hatte er nämlich vor der Lektüre des Tagebuches und kam danach zu der Überzeugung, dass diese Vorstellung »falsch war«. Denn: »Goebbels verachtete die übrige Menschheit, aber er verehrte Hitler mit unerschütterlicher Hingabe. Ungewöhnlich eitel und ehrgeizig, setzte er seine ganze machiavellistische Begabung für den Führer ein, der für ihn die >Mission der Deutschen $<$ verkörperte. $«^{21}$

Für Crossmann erklärte sich damit auch die Tatsache, die im Tagebuch besonders rätselhaft erschien. Denn für den Propagandaminister des >Dritten Reiches ‘ Goebbels war es geradezu eine Selbstverständlichkeit und absolut gerechtfertigt, »die Wahrheit« zu unterdrücken und zu verdrehen (S. 93). Sozusagen als exemplarisches Bei-

19 Ebd., S. 92. Goebbels’ »Verachtung « richtete sich auch auf den früheren Achsenpartner und Verbündeten Italien, denn: »Auch der Faschismus ist ein Betrug. Als Mussolini stürzt [...] ist Coebbels überrascht, aber kaum enttäuscht. Mussolinis Befreiung durch SS-Falschirmjäger im September 1943 beunruhigt ihn, wenn er sie auch ihrer psychologischen Auswirkungen wegen begrüßt. Deutschland kann durch sie um die Provinz Venetien gebracht werden, die sonst der Preis für den italienischen Verrat wäre.«(Ebd., S. 92)

20 Ebd., S. 92.

21 Ebd., S. $92 \mathrm{f}$. 
spiel für die »Einstellung zur Propaganda« von Goebbels stand für Crossmann der historische »Fall Katyn ${ }^{22}$.

In Anbetracht der Eintragungen von Goebbels in seinem Tagebuch, wo er sich über die >Lügen und die Unterdrückung « von »Reuter« und der »BBC«, deren sich beide Institutionen schuldig gemacht hatten, erregte, und nur in Bezug auf die persönlich verfassten Artikel in der nationalsozialistischen Zeitschrift Das Reich von »Offenheit und Objektivität« sprach, drängte sich für Crossmann die Frage auf: »Ist das nur Schauspielerei?« (Ebd.) Angesichts der Tatsache, dass Goebbels diese Ausführungen im Kontext zum »Fall Katyn« in seinem persönlichen geheimen Tagebuch gemacht hatte und seine Empörung »alle Zeichen einer geradezu hysterischen Aufrichtigkeit« (ebd.) trug, bestand für Crossmann kein Zweifel, dass Goebbels nicht als »Schauspieler « fungierte. Für ihn stand fest:

Tatsächlich liegen die Dinge so, daß die Nazis nicht aufgehört hatten, an Wahrheit und Anständigkeit zu glauben, sich selbst aber für solche Übermenschen im Sinne Nietzsches hielten, daß sie allein von allen Beschränkungen durch menschliche Gesetzte befreit waren. Goebbels hatte die »dekadenten bürgerlichen Werte« für Deutschland abgeschafft, aber er war der Meinung, daß die Demokratien sich so lange an sie halten sollten, bis sie ebenso zerstört waren wie die deutsche Republik. Deutschland war berechtigt, totalen Krieg zu führen, England nicht. Wenn Hitler einen Pakt mit Stalin unterzeichnete, so war das ein glänzender Schachzug [...]. ${ }^{23}$

Die Erkenntnis, die sich für Crossmann aus dem Studium des Tagebuches ergab, war darin zu sehen, zu verstehen: »wie der Übermensch sich selbst zerstört«. Denn, so der Autor: »Sich selbst >jenseits von Gut und Böse $\mathrm{zu}$ stellen, bedeutet letztlich, auch die Möglichkeit zu verlieren, Wahrheit und Lüge auseinanderzuhalten. So ist es Goebbels ergangen. Er begann als glänzender Propagandist und endete damit, daß er Objektivität als Hochverrat anprangerte und seine eigene Propaganda glaubte. Die Lüge auf seinen Lippen « - so beendete Crossmann seinen Beitrag Dr. Goebbels privat - „war zu einer Lüge seines ganzen Wesens geworden. ${ }^{24}$

\subsection{Die Genesis der nationalsozialistischen "Machtergreifung" oder: das "Scheitern « der Weimarer Republik.}

Damit die nationalsozialistische Bewegung und der »Führer« der NSDAP, Adolf Hitler, an die Schaltstellen der staatlichen Macht gelangen konnte, stand für die Zeitschrift Der Monat, genauer: für einige prominente Autoren, fest, dass der Erste Weltkrieg eine wichtige, um nicht zu sagen entscheidende historische Zäsur darstellte. Zweifelsohne sah man gerade in diesem Ereignis die »Urkatastrophe« (Kennan) für den weiteren Verlauf der europäischen Geschichte. Beispielsweise fällte Bondy - unter Berufung auf den Schweizer Historiker Jean Rudolf von Salis - mit Blick auf den sogenannten europäischen Bürgerkrieg das Urteil: »Das Jahr 1914 hatte einen Bruch bedeutet, dessen Schwere erst 1918 den europäischen Völkern ganz zum Bewußtsein kam, und des-

22 Siehe hierzu im Einzelnen ebd., S. 93.

23 Ebd.

24 Ebd. 